\title{
Overlapping cusp ion injections: An explanation invoking magnetopause reconnection
}

Article

Published Version

Lockwood, M. (1995) Overlapping cusp ion injections: An explanation invoking magnetopause reconnection.

Geophysical Research Letters, 22 (9). pp. 1141-1144. ISSN 00948276 doi: https://doi.org/10.1029/95GL00811 Available at https://centaur.reading.ac.uk/38804/

It is advisable to refer to the publisher's version if you intend to cite from the work. See Guidance on citing.

Published version at: http://dx.doi.org/10.1029/95GL00811

To link to this article DOI: http://dx.doi.org/10.1029/95GL00811

All outputs in CentAUR are protected by Intellectual Property Rights law, including copyright law. Copyright and IPR is retained by the creators or other copyright holders. Terms and conditions for use of this material are defined in the End User Agreement.

www.reading.ac.uk/centaur 
Central Archive at the University of Reading

Reading's research outputs online 


\title{
Overlapping cusp ion injections: An explanation invoking magnetopause reconnection
}

\author{
M. Lockwood \\ Rutherford Appleton Laboratory, Chilton, Oxfordshire, UK.
}

\begin{abstract}
An explanation of overlapping cusp ion injections is presented using the pulsating cusp model of the effects of magnetopause reconnection. It is shown that two populations of cusp ions, covering separated energy ranges, can be seen simultaneously by low- or mid-altitude satellites because of the combined effect of the acceleration and the straightening of newly-opened field lines as they evolve away from the reconnection site. Observations of such signatures, recently reported in data from the Viking and Freja satellites, are discussed in terms of pulsed and steady reconnection.
\end{abstract}

\section{Introduction}

Observations of magnetosheath-like ion populations precipitating in the cusp region have revealed that "overlapping injections" can sometimes occur. At any one time during such events, two plasma populations are detccted, with an energy gap between them in which no or few ions are seen. This was first reported by Carlson and Torbert [1980] from rocket observations, but recently has also been detected by the Viking satellite at mid-altitudes [Woch and Lundin, 1991; 1992; Yamauchi and Lundin, 1994] and the Freja satellite in the topside ionosphere [Norberg et al., 1994]. One way that such a feature could arise, discussed by Burch et al. [1982], is if significant numbers of heavier ions are injected along with the protons. In such cases, an ion of atomic mass $a$ (in amu) would have the same flight time as a simultaneously-injected proton if its energy was larger by the factor $a$. However, the data show cases which do not maintain this fixed energy ratio and mass-resolving ion detectors show that the overlap can occur for each ion species individually [Norberg et al., 1994]. Both spectrogram traces in an overlap event display both energy-latitude and energy-pitch angle dispersions, indicating that the particles are precipitating down convecting field lines. Lockwood and Smith [1994] suggested that some mid-altitude observations of these events were due to fully pulsed reconnection and a finite gyroradius effect. However, if this were to be the only cause, overlap events would be unlikely at low-altitudes where only ions injected with close to zero pitch angle can be detected. Hence this does not explain the low-altitude Frcja observations recently reported by Norberg et al. [1994].

Lockwood and Smith [1992; 1993; 1994] and Onsager [1994] have stressed that zero pitch-angle ions with different energies, seen simultaneously inside the magnetosphere/iono-

Copyright 1995 by the American Geophysical Union.

Paper number 95GL00811

0094-8534/95/95GL-00811\$03.00 sphere on a convecting field line, must have been injected across different points of the magnetopause. This is because different energy ions have different flight times from the magnetopause to the satellite. The spectrum seen at any one time (and thus on one field line) is an ensemble: ions of low energy are injected earlier and the field line moves before the higher energy ions enter. A key point about the overlapping events is that they reveal two regions of the magnetopause where ions enter the magnetosphere and reach the satellite, separated by a region where ions either do not cross the magnetopause or, if they do, they fail to reach the satellite.

There is much evidence that cusp particles enter the magnetosphere along newly-opened field lines produced by reconnection in the dayside magnetopause [see review by Lockwood and Smith, 1994]. However, it is not obvious how this can produce the overlapping signatures. The problem is that once a field line is opened, ions stream continuously across the boundary. It is difficult to devise a scenario in which a field line is opened, closed and then opened again as it convects. In this paper, however, it is shown that it is not necessary to modulate the particle entry in this fashion. Overlapping signatures can arise for the continuous particle entry predicted by the reconnection model, because of the variation of the ion acceleration on crossing the magnetopause, combined with the ion time-of-flight effects.

\section{Ion entry and acceleration predicted by the reconnection model}

Figure 1 shows the dayside magnetosphere in the open magnetosphere model, for southward IMF conditions. A newly-opened field line is shown in four positions (labelled $1-4)$ as it evolves away from the reconnection site, $X$, under the influence of the magnetic curvature force ("tension") and the magnetosheath flow. The points $P_{n}$ are where the field line (in position $n$ ) threads the magnetopause. Each and every newly-opened field line executes this sequence. A satellite $S$ at low altitudes is shown observing a field line in position 4 , where it sees a range of ion energies with the most energetic coming from $\mathrm{P}_{3}$ and the least energetic coming from $\mathrm{P}_{1}$, near the $\mathrm{X}$-line. At all $\mathrm{P}_{\mathrm{n}}$, ions stream across the magnetopause, as confirmed by stress-balance tests applied to both the dayside and the near-Earth tail boundaries [see Lockwood and Smith, 1994]. To get an overlapping signature, S must see ions from around $\mathrm{P}_{3}$ and $\mathrm{P}_{1}$ but not from around $\mathrm{P}_{2}$.

Figure $2 \mathrm{a}$ shows the general situation at the rotational discontinuity formed by the newly-opened field lines at a point $\mathrm{P}_{\mathrm{n}}$. In the $\mathrm{ZX}$ plane shown, the field line makes angles $\phi$ and $\theta$ with the magnetopause on its magnetosheath and magnetospheric sides, respectively. These angles increase with the distance from $X$ to $P_{n}$, as the field line straightens. Cowley [1982] derived a theory to predict D-shaped distribu- 


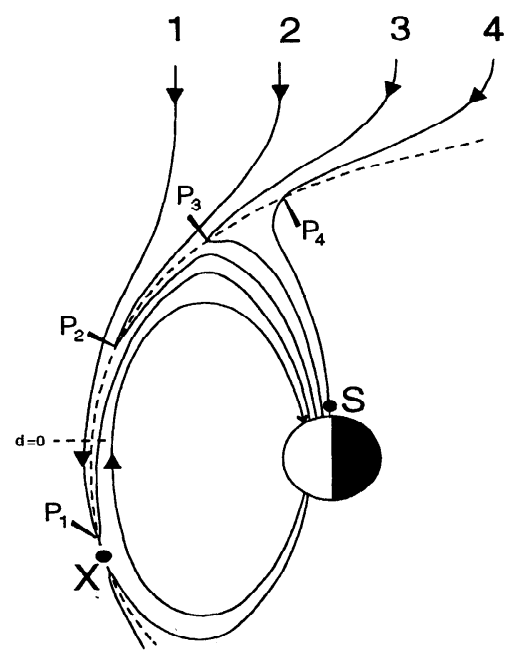

Figure 1. A schematic of the evolution of a newly-opened field line away from a low-latitude reconnection site $\mathrm{X}$.

tion functions of ions injected and accelerated across the boundary by flowing along such newly-opened field lines. These predictions have recently been verified in considerable quantitative and qualitative detail [Smith and Rodgers, 1991; Fuselier et al., 1991] and were made using constructions like that shown in Figure $2 \mathrm{~b}$. This is a velocity space plot in the Earth's frame $\left(V_{x}^{\prime}, V_{z}^{\prime}\right)$ and in the de-Hoffman Teller $\left(V_{x}, V_{z}\right)$ frame (hereafter referred to as the dHT frame). The dHT frame moves with the field line at velocity $V_{f}$ (in the Earth's frame) in the $+Z$ direction such that in it there is no electric field and hence ion energy is conserved on crossing the boundary. For the simplified symmetric case employed here, in which the Alfven speed $V_{A}$ is the same on both sides of the boundary, the only bulk ion motion in the dHT frame is field-aligned at the constant speed $V_{A}$ : sheath ions approaching the magnetopause have $V_{x}<0$ and $V_{z}<0$ (s in Figure $2 \mathrm{~b}$ ), but once injected they have $V_{x}<0$ but $V_{z}>0$ (i in Figure 2b). About this bulk flow is the thermal spread and only ions with $V_{x}<0$ will cross the magnetopause: the minimum injected field-aligned velocity in the dHT frame is zero and Figure $2 b$ shows that this gives a minimum fieldaligned velocity of $V_{f} \cos \theta$ in the Earth's frame. The fieldaligned bulk flow in the Earth's frame is $V_{p}=\left(V_{f} \cos \theta+V_{A}\right)$.
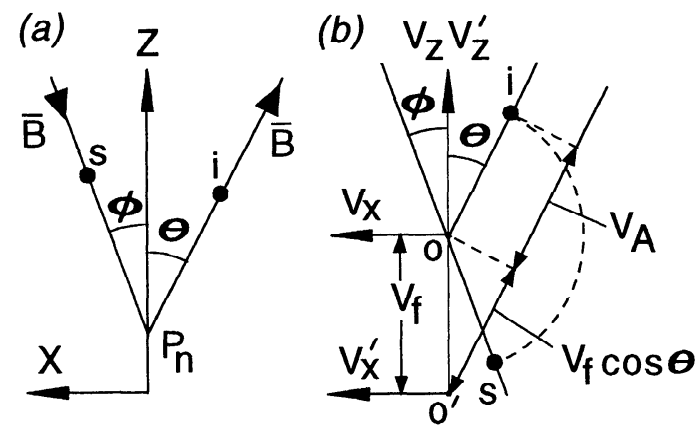

Figure 2. (a) The rotational discontinuity (RD) formed by a newly-opened field line at $\mathrm{P}_{\mathrm{n}}$ which evolves along the magnetopause in the $Z$ direction at speed $V_{f}$ in the Earth's frame of reference. The $\mathrm{X}$ direction is the outward normal to the boundary. (b) A velocity space plot showing field-aligned bulk flow at the Alfvén speed, $V_{A}$, in the de-Hoffman Teller frame $\left(V_{x}, V_{z}\right.$ with origin at $\mathrm{O}$ ) both into the RD in the magnetosheath (at $\mathrm{s}$ ) and away from the RD in the magnetosphere (at i). This geometric construction allows the calculation of the minimum and bulk flow field-aligned speed of injected particles in the Earth's frame $\left(V_{x}^{\prime}, V_{z}^{\prime}\right.$ with origin at $\left.\mathrm{O}^{\prime}\right)$. (Adapted from Cowley, [1982]).

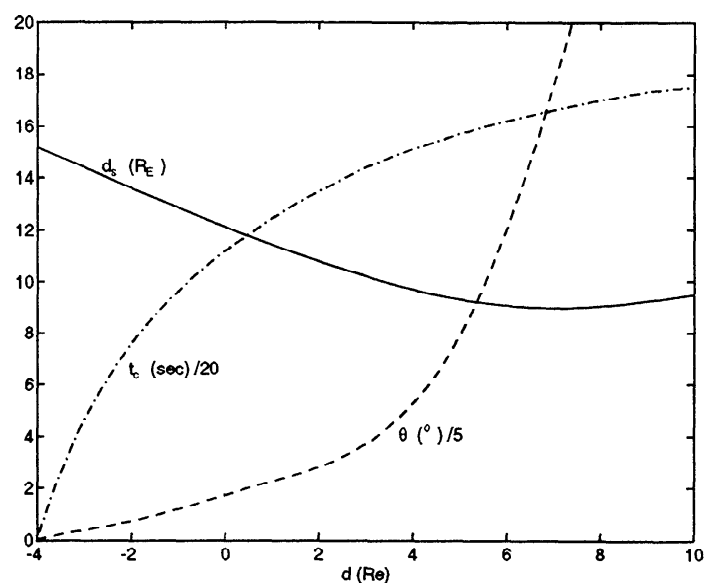

Figure 3. Variations of assumed model inputs with distance $d$ from the nose of the magnetosphere for an X-line at $d=-4 R_{E}$. The solid line is the distance $d_{s}$ from the injection point $\mathrm{P}_{\mathrm{n}}$ to the satellite (in units of $R_{E}$ ) and the dashed line is the angle $\theta / 5$, where $\theta$ is in degrees and is defined by Figure $2 \mathrm{a}$. The dot-dash line is $t_{c} / 20$, where $t_{c}$ is the time (in seconds) for the field line to convect from the $\mathrm{X}$ line to $\mathrm{P}_{\mathrm{n}}$ and is derived by the model.

Cowley and Owen [1989] have noted that $V_{f}$ is such that $V_{s h}$, the $\mathrm{X}$ component of the sheath flow which is tangential and adjacent to the boundary and in the Earth's frame, results in the field-aligned inflow speed in the dHT frame being $V_{A}$, so

$$
V_{f}=V_{s h}+V_{A} \cos \phi .
$$

Thus there is a minimum field-aligned energy of the population of injected ions (of mass $m$ ) of

$$
E_{\text {min }}=m\left(V_{f} \cos \theta\right)^{2} / 2=m\left(\left[V_{s h}+V_{A} \cos \phi\right] \cos \theta\right)^{2} / 2
$$

Lockwood and Smith [1994] pointed out that $E_{\min }$ firstly rises as the field line accelerates away from the $\mathrm{X}$-line $\left(V_{f}\right.$ increases), but then falls to zero as $\theta$ increases to $90^{\circ}$.

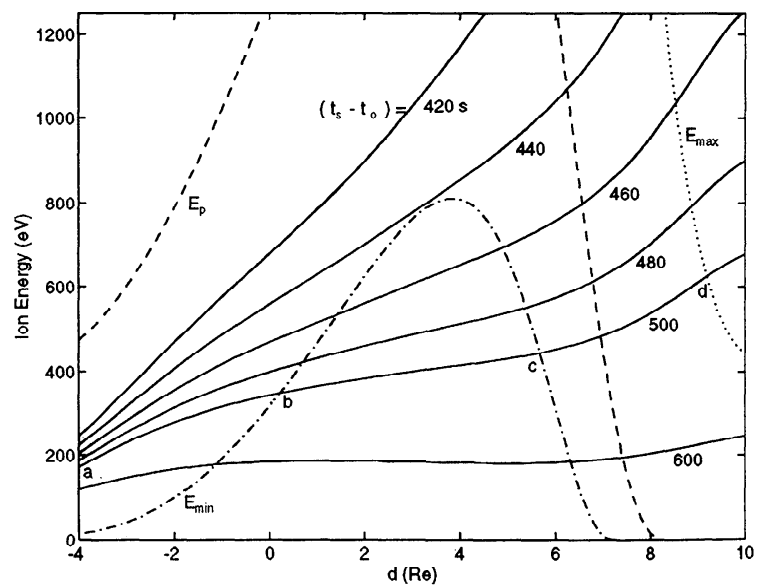

Figure 4. The ion energy, $E_{n}$, received from the magnetopause point $\mathrm{P}_{\mathrm{n}}$, as a function of $d$, the distance of $\mathrm{P}_{\mathrm{n}}$ along the magnetopause from the nose of the magnetosphere, for various clapsed times since reconnection $\left(t_{s}-t_{o}\right)$. The dot-dash, dashed and dotted lines are, respectively, the minimum energy $\left(E_{\min }\right)$, the energy of peak distribution function $\left(E_{p}\right)$ and the peak detected energy $\left(E_{\max }\right)$ injected at $\mathrm{P}_{\mathrm{n}}$. 


\section{An explanation of overlapping injections}

If at time $t_{s}$, we are observing a field line opened at a time $t_{o}$ and which took a time $t_{c}$ to evolve from $\mathrm{X}$ to an injection point $P_{n}$, then the ion time of flight from that $P_{n}$ to $S$ is

$$
t_{s}-\left(t_{o}+t_{c}\right)=d_{s} /\left(2 E_{n} / m\right)^{1 / 2}
$$

where $d_{s}$ is the field-aligned distance from the point $\mathrm{P}_{\mathrm{n}}$ to the satellite $\mathrm{S}$ and $E_{n}$ is the only energy of field-aligned ions which $\mathrm{S}$ can detect from the point $\mathrm{P}_{\mathrm{n}}$ at that $t_{s}$.

The argument used here is that, as the minimum injected ion energy of the Cowley D distribution rises and then falls, there can be a range of points $P_{n}$ on the magnetopause for which this minimum energy is larger than the only energy which could be seen by S (i.e. $E_{\text {min }}>E_{n}$ ), even though lower energies (from closer to the $\mathrm{X}$-line) and higher energies (from closer to the satellite) are both seen. This therefore gives the overlapping injection signature at any one observation time $t_{s}$. That this can occur is demonstrated here by a simple model calculation. To make the effect clearer, the reconnection X-line is beyond the magnetosheath stagnation region at the nose of the magnetosphere. As in Cowley and Owen [1989], magnetic effects on the sheath flow are neglected so that the tangential sheath velocity $V_{s h}$ can be taken from a gas-dynamic model [Sprieter et al., 1966] as a function of the distance $d$ from the nose of the magnetosphere (which is taken to be at a stand-off distance of $10 R_{E}$ ). The $\mathrm{X}$ line is placed at $d=-4 R_{E}$ (i.e., $4 R_{E}$ beyond the nose) and, for simplicity, we employ a highly draped sheath field such that $V_{A}$ and $\cos \phi$ are independent of $d$ and equal to 250 $\mathrm{km} \mathrm{s}^{-1}$ and 1 , respectively: assumed variations with $d$ of the distance $d_{s}$ and the angle $\theta$ are inputs to the model, given by the solid and dashed lines in Figure 3. From equation (1) we can then compute the field line velocity $V_{f}$ at all $d$ and hence the time $t_{c}$ as a function of $d$ : the result is shown by the dotdash line in Figure 3. Equation (3) gives the variations of $E_{n}$ with $d$, shown as solid lines in Figure 4 for field lines of various elapsed-time since reconnection, $\left(t_{s}-t_{0}\right)$. Also shown is: the variation of $E_{\min }$ (dot-dash line), calculated using equation (2); the energy of the distribution function peak, $E_{p}$, corresponding to the field-aligned ion speed $V_{p}$ (dashed line); and the peak detectable energy, $E_{\max }$ (dotted line). $E_{\max }$ depends on the instrument sensitivity and the magnetosheath densities, and is here crudely estimated by saying the detector can see distribution function $f(v)$ for $v$ up to $\left(V_{p}+500\right) \mathrm{km} \mathrm{s}^{-1}$. It can be seen that for $\left(t_{s}-t_{o}\right)>440 \mathrm{~s}$ there are points on the magnetopause for which $E_{\min }>E_{n}$ and so they contribute no ions to the spectrum seen by $\mathrm{S}$ at that $t_{s}$. For example, at $\left(t_{s}-t_{o}\right)=500 \mathrm{~s}$ ions will be seen on the segments ab and cd of the relevant $E_{n}(d)$ curve in Figure 4, but not on bc for which $E_{n}<E_{\min }$. Hence there is an energy range for which no ions are seen by $S$. This gap is largest for the $\left(t_{s}-t_{0}\right)=460 \mathrm{~s}$ case, but drops to zero width for $\left(t_{s}-t_{o}\right)=600 \mathrm{~s}$. Thus it is possible for two magnetopause injection regions to contribute ions to the spectrum seen at any one time, separated by a region which does not (even though the injection takes place at all points of the magnetopause). Figure 5 is a schematic of two over-lapping events such that at $t_{s}=t_{1}$ the satellite sees a field line which has an elapsed time since reconnection of $\left(t_{s}-t_{o}\right)=500 \mathrm{~s}:$ the points a-d are marked. The evolution of these points with $t_{s}$ is discussed in the following section.

\section{Explaining observed overlapping injections using pulsed and steady reconnection}

Norberg et al. [1994] argue that only downward cusp ion steps can be produced by pulsed reconnection and, indeed, the papers they cite do only deal with such cases. However, Lockwood and Smith [1994] have shown that the nature of the step depends on the ratio of the components of the satellite motion and convection $\left(V_{s}\right.$ and $V_{c}$, respectively) which are normal to, and away from, the open/closed field line separatrix. In particular, they demonstrate that if $0<V_{s}<V_{c}$, the most common signature of pulsed reconnection will be downward ramps of ion energy with time, interrupted by upward steps, of the type presented by Norberg et al. This situation will often prevail for satellites like Viking, which follow a meridian at mid-altitudes, and for satellites like Freja, which move longitudinally at low altitudes (making the component $V_{s}$ small). Effectively, what happens is that the elapsed time since reconnection $\left(t_{s}-t_{o}\right)$ increases within each patch of newly-opened flux as it ages, so that the $E_{n}$ for all $\mathrm{P}_{\mathrm{n}}$ fall. However, the next patch of newly-opened flux (produced by the subsequent reconnection pulse) can be convected over the satellite (because $V_{s}<V_{c}$ ), giving a sudden fall in $\left(t_{s}-t_{o}\right)$ and a rise in $E_{n}$ for all $\mathrm{P}_{\mathrm{n}}$. On the other hand, low-altitude satellites moving meridionally poleward see only downward cusp ion steps because $V_{s} \gg V_{c}$.

Lockwood and Smith [1994] point out that an overlapping injection feature can be seen by a mid-altitude satellite about an upward cusp ion step, provided the reconnection only takes place in pulses, with zero reconnection rate between them. This is a finite ion gyro-radius effect and can be identified because the overlap will be present for larger pitch angle ions but downward-precipitating, field-aligned ions will only show the upward step with no overlap. Careful inspection of some of the cases reported from the Viking data reveals that they exactly fit this prediction. For example, in the lower panel of Figure 2 of Yamauchi and Lundin [1994] field-aligned ions are only seen at one energy at a time and that this energy steps up from the first to the second trace around 13:54:30. This is powerful cvidence that the reconnection rate fell to zero between two pulses.

The mechanism presented in the previous section will generate overlapping signatures in ions of all pitch angles,

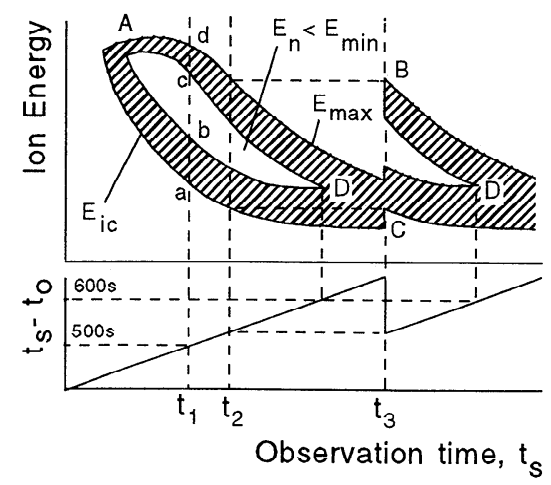

Figure 5. Schematic of two types of possible overlapping ion events, showing contours of the minimum detectable flux, such that cusp ions are only seen in the shaded regions. The reconnection is steady, except for a period of zero rate causing the upward step at $t_{s}=t_{3}$ : the lower panel gives the variation of the time elapsed since reconnection $\left(t_{s}-t_{0}\right)$. The points a-d and A$D$ are defined in the text with reference to Figure 4. 
including field-aligned. However, we also need to explain the observed sequences of cusp ion spectra with $t_{s}$. For $t_{s}<t_{3}$ in Figure 5 the reconnection is steady, so the poleward-moving satellite observes field lines of increasing $\left(t_{s}-t_{o}\right)$ with time $t_{s}$ and the minimum observed energy falls continuously (this time-of-flight cut-off energy was referred to by Lockwood and Smith [1992; 1994] as $E_{i c}$ and is $E_{n}$ for the X line). For the case shown in Figure 4, a clear overlapping signature will be seen after $\left(t_{s}-t_{o}\right)$ reaches $440 \mathrm{~s}$, when the distribution function $f(E)$ goes to zero at $800 \mathrm{eV}$ (because $E_{n}$ falls below $\left.E_{\text {min }}\right)$. In fact, consideration of the full Cowley-D distribution injected at each $\mathrm{P}_{\mathrm{n}}$ predicts a deepening minimum in $f(E)$, descending in energy, will have been observed before this as $\left(t_{s}-t_{o}\right)$ increases from about 410 to $440 \mathrm{~s}$. Thus the onset of the overlap would appear as a bifurcation of the pre-existing cusp signature ( $\mathrm{A}$ in Figure 5). Some observed examples could be interpreted as forming via such a bifurcation (for example, shortly after 00:53 in Figure 3 of Woch and Lundin [1991] is one possibility) and can thus be explained by steady reconnection and steady magnetosheath conditions. As discussed earlier, the width of the null reduces to zero at about $180 \mathrm{eV}$ when $\left(t_{s}-t_{o}\right)$ increases to about $600 \mathrm{~s}$. This steady-state case can thus predict the observed amalgamation of the two overlapping traces at low energies (the points D in Figure 5) [e.g. Figure 1 of Norberg et al., 1994].

In many other cases, however, (for example at 08:40:30 in Figure 7 of Lundin and Woch [1991] and 20:26:15 in Figure 1 of Norberg et al., [1994]) the higher energy, overlapping trace is, even at onset, detached in energy from the lowerenergy trace. This is not consistent with the bifurcation at $\mathrm{A}$ in Figure 5, but more like the onset B. There are two ways in which this could come about, both involving departures from steady state. In the first, there could be pulsed reconnection with $V_{c}>V_{s}$, such that an upward cusp ion step is formed. This occurs in Figure 5 at $t_{s}=t_{3}$ when $\left(t_{s}-t_{o}\right)$ discontinuously steps back to its value at $t_{2}$, giving an onset of a detached overlapping trace at $\mathrm{B}$. However, the lower trace would also show an upward step, including a step in $E_{i c}$, at C. This can be seen shortly after 00:53 in Figure 3 of Woch and Lundin [1991], but is not a general feature of the reported examples. Indeed, there are several cases of a detached onset of the upper trace, with no apparent step in the dispersion of the lower trace (i.e B without C) [e.g. Figure 1 of Norberg et al., 1994; Figure 7 of Woch and Lundin, 1991]. These therefore appear to be cases where the reconnection rate is relatively steady (meaning that rate variations are smaller than a factor of about 2 [Lockwood and Smith, 1994, Lockwood et al., 1994]) at a static X-line. In such cases, the appearance of the overlapping signature could be explained by a rapid fall in the magnetopause Alfvén speed (caused by a fall in the magnetic field and/or a rise in the plasma density). This would cause a fall in the field line speed $V_{f}$ and in the ion acceleration on crossing the magnetopause with reconnection time $t_{o}$. Thus $E_{\min }, E_{p}$ and $E_{\max }$ would all rise suddenly for all $\mathrm{P}_{\mathrm{n}}$ with increasing $\left(t_{s}-t_{o}\right)$. As the satellite observed field lines of higher $\left(t_{s}-t_{o}\right)$, the sudden onset of an overlap (but with no step in $E_{i c}$ ) could be seen as it moved onto earlier-reconnected (smaller $t_{o}$ ) field lines for which the acceleration was higher. Several events appear to fall into this class and quasi-steady reconnection and magnetosheath changes may be a common cause.

It has been shown here that the continuous entry of magnetosheath ions, along newly-opened field lines produced by magnetopause reconnection, can give rise to "overlapping injection" signatures of cusp ions due to the acceleration and straightening of the field lines. The model calculations presented will not match all such observations in all details, due to the simplifying assumptions made. The model also provides an explanation of why overlapping signatures are not always seen. This is because $E_{\min }$ may never exceed $E_{n}$, for example if $d_{s}$ is large because the magnetosphere is less compressed because the solar wind dynamic pressure is low.

Acknowledgements. ML is supported by the UK Particle Physics and Astronomy Research Council.

\section{References}

Burch, J.L, Plasma injection and transport in the mid-altitude polar cusp, Geophys. Res. Lett., 9, 921-924, 1982.

Carlson, C.W. and R.B. Torbert, Solar wind injections in the morning auroral oval, J. Geophys. Res., 85, 2903-2908, 1980.

Cowley, S.W.H and C.J. Owen, A simple illustrative model of open flux tube motion over the dayside magnetopause, Planet. Space Sci., 37, 1461, 1989.

Cowley, S.W.H., The causes of convection in the Earth's magnetosphere: A revicw of developments during IMS, Rev. Geophys., 20, 531, 1982.

Fuselier, S.A., D.M. Klumpar, and E.G. Shelley, Ion reflection and transmission during reconnection at the Earth's subsolar magnetopause, Geophys. Res. Lett., 18, 139, 1991.

Lockwood, M., and M.F. Smith, The variation of reconnection rate at the dayside magnetopause and cusp ion precipitation, J. Geophys. Res., 97, 14,841, 1992.

Lockwood and M.F. Smith, Comment on "Mapping the dayside ionosphere to the magnetosphere according to particle precipitation characteristics" by Newell and Meng, Geophys. Res. Lett., 20, 1739-1740, 1993

Lockwood, M., and M.F. Smith, Low- and mid-altitude cusp particle signatures for general magnetopause reconnection rate variations: I - Theory, J. Geophys. Res., 99, 8531-8555, 1994.

Lockwood, M., et al., The characteristics of the magnetopause reconnection $\mathrm{X}$-line deduced from low-altitude satellite observations of cusp ions, Geophys. Res. Lett., in press, 1994.

Norberg O., et al., Freja observations of multiple injection events in the cusp, Geophys. Res. Lett., 21, 1919-1922, 1994.

Onsager, T.G., A quantitative model of magnetosheath plasma in the low-latitude boundary layer, cusp and mantle in "Physical signatures of magnetopause boundary layer Processes", ed. J.A. Holtet and A. Egeland, NATO ASI Series $C$, Vol. 425, Kluwer, 385-400, 1994.

Smith, M.F. and Rodgers, D.J., Ion distributions at the dayside magnetopause, J. Geophys. Res., 95, 11,617, 1991.

Spreiter, J.R. et al., Hydromagnetic flow around the magnetosphere, Planet. Space Sci., 14, 223-253, 1966.

Woch, J., and R. Lundin, Temporal magnetosheath plasma injection observed with Viking: a case study, Ann. Geophys., 9, 133-142, 1991

Woch J., and R. Lundin, Signatures of transient boundary layer processes observed with Viking, J. Geophys. Res., 97, 14311447, 1992.

Yamauchi, M. and R. Lundin, Classification of large-scale and meso-scale ion dispersion patterns seen by Viking over the cusp-mantle region, in "Physical signatures of magnetopause boundary layer Processes", ed. J.A. Holtet and A. Egeland, NATO ASI Series C, Vol. 425, Kluwer, 385-400, 1994.

M. Lockwood, RAL, Chilton, Didcot, OX11 0QX, UK. (email: Internet.mike@eiscat.ag.rl.ac.uk)

(Received: November 7, 1994; revised, January 20, 1995; accepted: February 16, 1995.) 